ARTICLE OPEN

\title{
Trophic networks improve the performance of microbial anodes treating wastewater
}

\author{
Christin Koch $\mathbb{D}^{1,4 *}$, Katharina J. Huber $\mathbb{D}^{2}$, Boyke Bunk $\mathbb{D}^{2}$, Jörg Overmann $\mathbb{D}^{2,3}$ and Falk Harnisch $\mathbb{D}^{1 *}$
}

Microbial anodes represent a distinct ecological niche that is characterized mainly by the terminal electron acceptor, i.e., the anode potential, and the substrate, i.e., the electron source. Here, we determine the performance and the biofilm community of anode microbiomes while using substrates of increasing complexity (organic acids or organic acids and sugar or real domestic wastewater) to mimic different, practically relevant, trophic levels. a-Diversity values increased with substrate complexity. In addition, the higher abundance value of Deltaproteobacteria in the biofilms corresponds to higher reactor performance (i.e., COD removal, current density, and Coulombic efficiency). In reactors exploiting real wastewater, the diversity of the planktonic microorganisms was only little affected. Microbiome network analysis revealed two important clusters for reactor performance as well as performanceindependent pathogen-containing clusters. Interestingly, Geobacter was not found to be integrated in the network underlining its outstanding individual ecological role in line with its importance for the efficiency of the electron harvest for all reactors. The microbiome analysis of different trophic levels and their temporal development from initial colonization to stable treatment demonstrate important principles for the implementation of microbial anodes for wastewater treatment.

npj Biofilms and Microbiomes (2019)5:27

; https://doi.org/10.1038/s41522-019-0100-y

\section{INTRODUCTION}

Complex microbial communities in technical systems, the socalled reactor microbiomes, form the foundation of environmental biotechnology. Wastewater treatment plants (WWTP) represent the most prominent and best established type of this technology. ${ }^{1}$ Microbiomes in WWTP transform nitrogen, carbon, and phosphorous compounds, and thereby enable sustainable water purification. $^{2}$ Due to their diversity, stability, and flexibility, reactor microbiomes form an unprecedented microbial resource for WW treatment and valorization. Domestic and industrial WW treatment is usually performed in aerobic and anaerobic phases for the oxidation of organic compounds and the denitrification, respectively, of which aeration is the most energy-demanding process. This high-energy demand of aerobic treatment is caused by the high chemical energy content of domestic WW of about $7 \mathrm{~kJ} \mathrm{~L}^{-1}$, and hence the requirement of sufficient oxygen supply for its microbial oxidation. ${ }^{3}$

Microbial electrochemical technologies (MET) offer a sustainable alternative for WW treatment, as they allow combining the oxidation of organic compounds with the generation of electricity, ${ }^{4}$ and thus utilization of the high chemical energy content of domestic WW. In general, MET provide a technology platform that links microbial metabolism, specifically the turnover of metabolic redox carriers like $\mathrm{NAD}(\mathrm{P}) \mathrm{H} / \mathrm{H}^{+}$or quinones, with the flow of electrons to or from an electrode. ${ }^{5,6}$ In recent years, a plethora of MET-based applications ranging from biosensors via water desalination to the synthesis of chemicals has been evolved. ${ }^{7,8}$ The most mature field of application for MET is the treatment and valorization of WW for electric power harvest and hydrogen production; first demonstration units are in the cubic-meter scale. ${ }^{9,10}$
In these MET reactors, anode microbiomes are the key component. Microorganisms oxidize the organic and inorganic WW constituents and transfer the liberated electrons to the anode which serves as a solid terminal electron acceptor (TEA). This extracellular electron transfer (EET) can take place by direct and indirect means $s^{4,11}$ and was extensively studied not only in pure cultures of members of the Geobacteraceae (e.g., Geobacter sulfurreducens $\mathrm{PCA}^{\mathrm{T}}{ }^{12}$ ) and of the Shewanellaceae (e.g., Shewanella oneidensis MR-1 ${ }^{\top}{ }^{13}$ ) but also in several microbiomes. ${ }^{11,14}$ Direct EET requires physical contact of the microorganisms whereas the indirect EET does not. ${ }^{15}$ Accordingly, the two types of transfer can be assumed to occur preferentially in biofilms or in planktonic cultures, respectively.

The anode compartment of a MET reactor provides a distinct ecological niche mainly characterized by two features. First, the anode serves as TEA for anaerobic respiration via EET. Second, the carbon and energy sources determine the actual carbon and redox reactions at the anode. Whereas the electrode potential can be controlled and is usually set to values between -200 and $+400 \mathrm{mV}$ vs. the standard hydrogen electrode (SHE), the types of carbon and energy sources in WW are highly variable and diverse in terms of chemical composition and concentration. Often, the specific composition cannot be determined and only sum parameters are assessed. The most prominent sum parameter is the chemical oxygen demand (COD), a measure for the oxygen equivalents (and hence the electrons) needed for the oxidation of all WW compounds to $\mathrm{CO}_{2}$. Thus, the $\mathrm{COD}$ is also the foundation for legal regulations. For instance, a COD $\leq 200 \mathrm{mg} \mathrm{L}^{-1}$ is required in Germany for treated WW to be released into the environment. ${ }^{16}$

The reactor microbiome of a MET reactor anode compartment for WW treatment has two major functionalities: first, cleaning WW

\footnotetext{
${ }^{1}$ Helmholtz-Centre for Environmental Research, Department of Environmental Microbiology Permoserstraße 15, 04318 Leipzig, Germany. ${ }^{2}$ Leibniz Institute DSMZ—German Collection of Microorganisms and Cell Cultures, Inhoffenstraße 7B, 38124 Braunschweig, Germany. ${ }^{3}$ Department of Life Sciences, Braunschweig University of Technology, Braunschweig, Germany; ${ }^{4}$ Present address: Global Innovation Cosmetic Ingredients, Symrise AG, Mühlenfeldstraße 1, 37603 Holzminden, Germany. ${ }^{*}$ email: christin.koch@symrise. com; falk.harnisch@ufz.de
} 
by oxidation of substrates (removal of COD), second, harvesting the electrons of the oxidation process as electric current by employing the anode as TEA. As electroactive microorganisms are metabolically limited, ${ }^{14,17}$ the challenge of MET engineering is to provide an ecological niche at the anode that fosters COD removal as well as electric current production by building up a wellbalanced food web.

For the translation into application, a better understanding of anode colonization and the development of the microbiome under real WW conditions is required. This includes, for instance, understanding the relationship between microbiome composition and functionality in terms of COD removal, current density (j), and electron efficiency (Coulombic efficiency, CE). Only very little is known of the ecological principles for the formation and temporal development of microbiomes in MET. Therefore, this study aims to shed light on the structure-function relationship of microbial anodes from initial colonization based on domestic WW to its stable treatment. To distinguish and understand the impact of the different trophic levels on COD removal and current production, WW of different complexity ranging from organic acids to real domestic WW was studied in eleven $500 \mathrm{~mL}$ reactors, run in parallel and inoculated identically. Electrochemical performance was continuously monitored and WW-related parameters as well as the microbiome composition of all anodic biofilms and planktonic phases were analyzed for each batch. This approach allowed us to determine the patterns of initial electrode colonization, the development of complex trophic networks, as well as of the key players for COD removal and electric current generation, and to derive general recommendations on the design and steering of anode microbiomes in WWTP.

\section{RESULTS}

Reactor performance

The Real_WW reactors were started without prior enrichment of electroactive biofilms with real domestic WW as the only source of inoculum and substrate. The WW was collected from a local WWTP on average characterized by the following parameters: COD $473 \pm$ $74 \mathrm{mg} \mathrm{L}^{-1}, \mathrm{pH} 8.0 \pm 0.1$, total nitrogen $89 \pm 6 \mathrm{mg} \mathrm{L}^{-1}$, ammonium $73 \pm 27 \mathrm{mg} \mathrm{L}^{-1}$, sulfide $0.9 \pm 0.4 \mathrm{mg} \mathrm{L}^{-1}$, and total organic carbon (TOC) $163 \pm 17 \mathrm{mg} \mathrm{L}^{-1}$. $^{18}$ All Real_WW reactors started current production already in batch I (Fig. 1) with a maximum current density of $0.04 \mathrm{~mA} \mathrm{~cm}^{-2}$ in Real_WW reactor 4 at day 5. Small cyclic variations in the current density are due to diurnal temperature changes. After each batch, the treated WW was completely removed from the reactor; the reactor was then refilled with fresh deaerated real WW and electrochemically incubated for another week. Cells attached to the electrodes and the glass walls of the reactor vessel remained within the reactor during the exchange. In general, the treated WW appeared more transparent then the fresh WW, but the formation of bigger flocks was observed.

Considering all six batches, the Real_WW reactors showed a similar performance regarding treatment efficiency $(\triangle C O D=55 \pm$ $18 \%)$ and electrochemical performance $(q=351 \pm 211 \mathrm{C}, \mathrm{CE}=$ $21 \pm 12 \%$ ), with individual exceptions especially for Real_WW reactors 1 and 2. Real_WW reactor 1 showed the lowest performance during the batches I-IV, but then it suddenly increased in current density during batch $\mathrm{V}$. This increase was also reflected in the COD removal efficiency that increased from an average of $30 \pm 6 \%$ during batch I-IV to $47 \%$ and $43 \%$ in batches $\mathrm{V}$ and $\mathrm{Vl}$, respectively. In contrast, Real_WW reactor
A

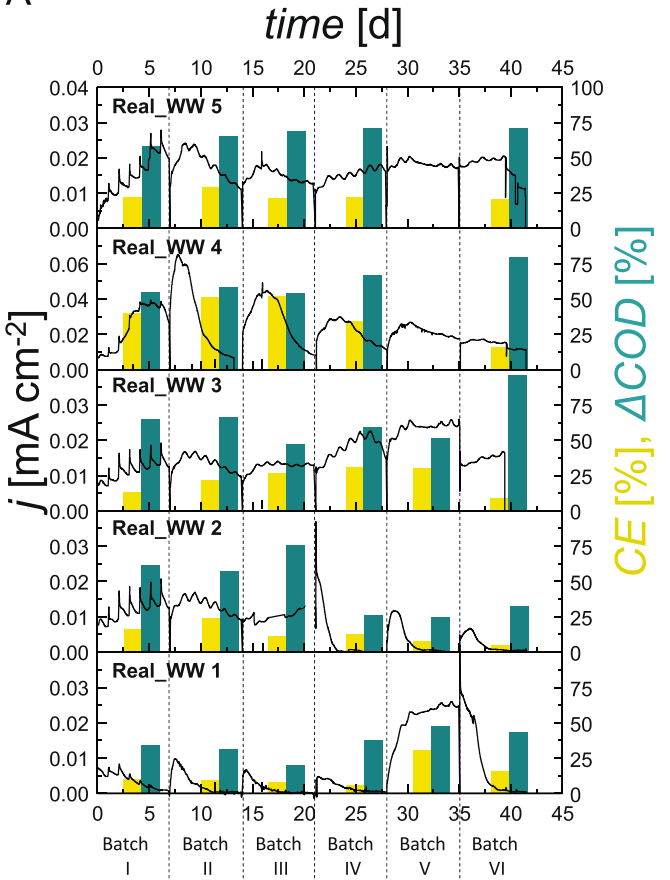

B

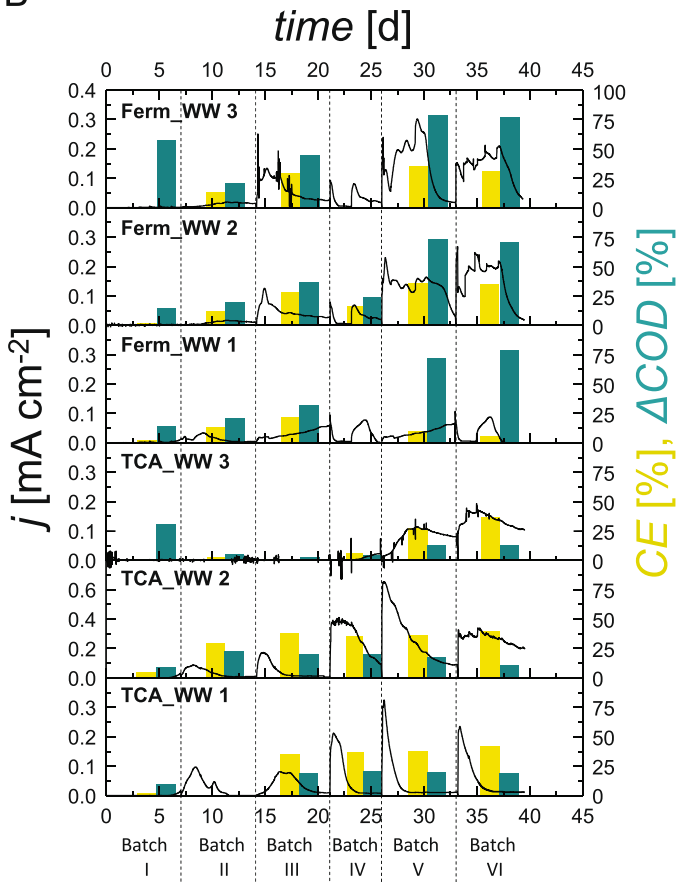

Fig. 1 Course of current density (j) and characteristic process parameters (Coulombic efficiency (CE, yellow) and COD removal ( $\triangle C O D$, turquoise)) in real wastewater reactors Real_WW 1-5 (a) and defined wastewater reactors TCA_WW 1-3 and Ferm_WW 1-3 (b). The current density (black line) was continuously monitored for all reactors over the course of the experiment, while the other parameters (colored bars) were determined per batch (detailed values in Supplementary Table 1). Note the different scaling of axis for $\mathbf{a}$ and $\mathbf{b}$ to allow the visualization of differences between reactors. The Real WW $1-5$ reactors were run with real domestic wastewater as the only source of carbon and microorganisms. The TCA_WW 1-3 and Ferm_WW 1-3 reactors were inoculated with 5\% real domestic WW in the first batch and run all batches with $0.6 \mathrm{~g} \mathrm{~L}^{-1}$ COD equivalents of propionate, butyrate and acetate (TCA_WW 1-3) or sucrose, and propionate, butyrate, and acetate (Ferm_WW 1-3). After each batch, the reactor liquid was completely replenished by fresh real domestic WW, respectively, defined as wastewater 


\begin{tabular}{|c|c|c|c|c|}
\hline & Sucrose & Acetate & Propionate & Butyrate \\
\hline \multicolumn{5}{|c|}{ TCA_WW reactors 1-3 } \\
\hline Batch I & - & $-0.1 \pm 0.5$ & $-0.7 \pm 0.4$ & $-0.7 \pm 0.4$ \\
\hline Batch II & - & $-1.6 \pm 1.3$ & $-0.3 \pm 0.2$ & $-0.2 \pm 0.0$ \\
\hline Batch III & - & $-1.4 \pm 1.2$ & $-0.5 \pm 0.3$ & $-0.1 \pm 0.1$ \\
\hline Batch IV & - & $-1.7 \pm 1.3$ & $-0.4 \pm 0.2$ & $-0.2 \pm 0.1$ \\
\hline Batch V & - & $-2.0 \pm 0.5$ & $-0.5 \pm 0.2$ & $-0.2 \pm 0.1$ \\
\hline Batch VI & - & $-1.7 \pm 0,4$ & $-0.5 \pm 0.3$ & $-0.0 \pm 0.2$ \\
\hline \multicolumn{5}{|c|}{ Ferm_WW reactors $1-3$} \\
\hline Batch I & $-2.0 \pm 0.0$ & $4.1 \pm 3.8$ & $1.7 \pm 0.6$ & $0.1 \pm 0.0$ \\
\hline Batch II & $-2.0 \pm 0.0$ & $6.4 \pm 0.4$ & $1.1 \pm 0.2$ & $0.1 \pm 0.0$ \\
\hline Batch III & $-2.0 \pm 0.0$ & $3.6 \pm 1.0$ & $1.3 \pm 0.4$ & $-0.0 \pm 0.0$ \\
\hline Batch IV & $-2.0 \pm 0.0$ & 6.0 & 0.9 & 0.2 \\
\hline Batch V & $-2.0 \pm 0.0$ & $-1.0 \pm 0.0$ & $0.5 \pm 0.2$ & $0.0 \pm 0.0$ \\
\hline Batch VI & $-2.0 \pm 0.0$ & $-1.0 \pm 0.0$ & $0.5 \pm 0.2$ & $-0.1 \pm 0.1$ \\
\hline \multicolumn{5}{|c|}{$\begin{array}{l}\text { Negative values represent a degradation compared with the start } \\
\text { concentration. Positive values indicate that the concentration of the } \\
\text { compound after a batch was higher than the initial concentration of the } \\
\text { batch, e.g., as a result of the fermentation of sucrose to organic acids. The } \\
\text { values give average } \pm \text { standard deviation in } \mathrm{mM}(n=3) \text {, except for } \\
\text { Ferm_WW batch IV }(n=1)\end{array}$} \\
\hline
\end{tabular}

2 showed a performance in line with reactors 1-3 over the first three batches $(q=316 \pm 57 \mathrm{C}, \Delta \mathrm{COD}=65 \pm 8 \%)$ and then declined significantly in batches IV-VI $(q=67 \pm 17 \mathrm{C}, \Delta \mathrm{COD}=$ $28 \pm 3 \%)$. Nevertheless, a successful COD removal, being the main measure for WW treatment, and current generation was found in all reactors (details in Fig. 1, Supplementary Table 1), and similar characteristics of the treated WW were determined with pH $7.1 \pm$ 0.3 , total nitrogen $86 \pm 9 \mathrm{~g} \mathrm{~L}^{-1}$, ammonium $14 \pm 2 \mathrm{~g} \mathrm{~L}^{-1}$, and TOC $107 \pm 32 \mathrm{mg} \mathrm{L}^{-1}$. Only the sulfide concentration differed ranging from below $0.5 \mathrm{~g} \mathrm{~L}^{-1}$ in Real_WW reactors 1 and 2 to above $20 \mathrm{~g}$ $\mathrm{L}^{-1}$ for the other reactors.

The reactors with defined WW (Ferm_WW and TCA_WW) were started with 5\% real WW as inoculum in batch I. No prior enrichments and no additional inoculation were performed in consecutive batches. In contrast to the Real_WW, all defined WW reactors showed increased performance over time. While maximum current densities in the first batch only reached $0.03 \mathrm{~mA}$ $\mathrm{cm}^{-2}$ (TCA_WW reactors 2 and 3 ) and the average charge was $q=$ $40 \pm 15 C$, these values increased significantly in the following batches with the highest values in batches V and VI. Here, the maximum current densities were one order of magnitude higher than that for Real_WW (e.g., $j=0.30 \mathrm{~mA} \mathrm{~cm}^{-2}$ batch V, TCA_WW reactor 3 and $j=0.03 \mathrm{~mA} \mathrm{~cm}^{-2}$ batch $\mathrm{V}$, Real_WW reactor 4). The accumulated charge was about three times higher for the defined WW with Ferm_WW 1-3 $(q=2178 \pm 1134 C$, batch VI) for WW based on organic acids and sugar compared with TCA_WW 1-3 $(q=773 \pm 224 \mathrm{C}$, batch VI) exploiting WW based on organic acids only. While the charge production $(q=803 \pm 369$ C for TCA_WW and $q=1731 \pm 1389 C$ for Ferm_WW) and COD removal $(\triangle C O D=$ $15 \pm 5 \%$ for TCA_WW, $\triangle C O D=68 \pm 5 \%$ for Ferm_WW) differed significantly between the two types of defined WW $(p=0.03$ and $p=0.00$, student's $t$ test considering batches IV-VI), this difference was not found for the CE $(p=0.10)$ being $33 \pm 10 \%$ and $24 \pm 12 \%$ (batches IV-VI).

While the individual degradation pathways for the complex substrate composition in domestic WW are difficult to determine, the fate of individual substrates was studied by using the defined WW. The concentrations of sucrose (only in Ferm_WW) and acetate, propionate, and butyrate (in TCA_WW and Ferm_WW) were determined after each batch (Table 1). In general, the substrates were not only degraded bioelectrochemically, as the maximum CE was only $42 \%$. Ferm_WW sucrose was always completely removed. The main share of the primary degradation was most likely performed by planktonic cells as only in Ferm_WW $1-3$, the planktonic phase was turbid and showed a visible biomass increase. As a result of the sucrose conversion, an accumulation of acetate and propionate was found in batches I-IV. Nevertheless, the partial degradation of the organic acids resulted in an average COD removal over all batches of $15 \pm 7 \%$ (TCA_WW 1-3) and $46 \pm 25 \%$ (Ferm_WW 1-3). Further, a reddish biofilm formation at the anodes was observed in all reactors.

Bacterial community analysis

All reactors were started by using the identical real domestic WW inoculum at the identical point of time. Clear differences in the electrochemical performance were observed for the different reactor setups containing either Real_WW or a TCA_WW and Ferm_WW. Yet, also differences in the performance of the parallelrun reactors were evident (see the section above). These differences are most likely the result of stochastic biological processes, ${ }^{19,20}$ as shown previously also for the colonization of anodes. ${ }^{21}$ Therefore, the primary colonization of the anodes from the domestic WW inoculum was determined and followed in its development over time.

The real WW had a stable community composition over six batches (60-70\% Proteobacteria, 11-13\% Firmicutes, and 12-19\% Bacteroidetes) although it was freshly collected every week (complete data set with relative abundance data of all genera in all samples is provided as Supplementary Data 1). It was further dominated by the genus Arcobacter (Epsilonproteobacteria) to which $47 \pm 3 \%$ of all sequences were assigned. Arcobacter has been found in numerous other environmental samples, i.e., estuarine sediment, marine water, ${ }^{22,23}$ but its functional relevance and the reason for its dominance in these WW reactors are not known. The community composition of the treated WW (planktonic phase) in the Real_WW reactors $3-5$ is highly similar to the original WW and only little variation was found over the different batches. Real_WW reactors 1 and 2 possess a higher variability in the community composition of the treated WW but show similar trends. The contribution of Arcobacter varies in the treated real WW samples between $1 \%$ and $48 \%$.

The biofilm samples of the Real_WW reactors 1-5 clearly differ from the inoculum and the planktonic phase (Fig. 2). This shows a specific enrichment of a functional bacterial community by growth on the anode surface rather than a random attachment of bacterial cells. The biofilm samples show a similar composition on the genus level, but differ in the individual abundances with similar trends over time. Especially, the enrichment of Deltaproteobacteria became obvious in all biofilms starting with $34 \pm 10 \%$ in batch II and increasing to $45 \pm 12 \%$ in batch Vl. Eight different genera were assigned to the Deltaproteobacteria (Fig. 3) with Geobacter having the highest contribution over all samples with a relative abundance of $22 \pm 14 \%$. Especially in the Real_WW reactors 4 and 5, Geobacter clearly dominated the bacterial community with $34 \pm 9 \%$ (Real_WW reactor 4) and $36 \pm 10 \%$ (Real_WW reactor 5). The second most abundant Deltaproteobacteria are Desulfobacter $(9 \pm 5 \%)$ and Desulfuromonas ( $1 \pm 6 \%)$. These two genera are known for sulfate and sulfur reduction ${ }^{24}$ and Desulfuromonas even has an electroactive representative. ${ }^{17}$

The anode biofilms of the Ferm_WW reactors 1-3 and TCA_WW reactors 1-3 also clearly differed from the inoculum and clustered closer to the anode biofilms of the Real_WW reactors (Fig. 2). The anode biofilms of the defined WW reactors also show a high enrichment of Deltaproteobacteria with $45 \pm 22 \%$. Geobacter seems to be an efficient first colonizer with $25 \pm 12 \%$ in batch II. 


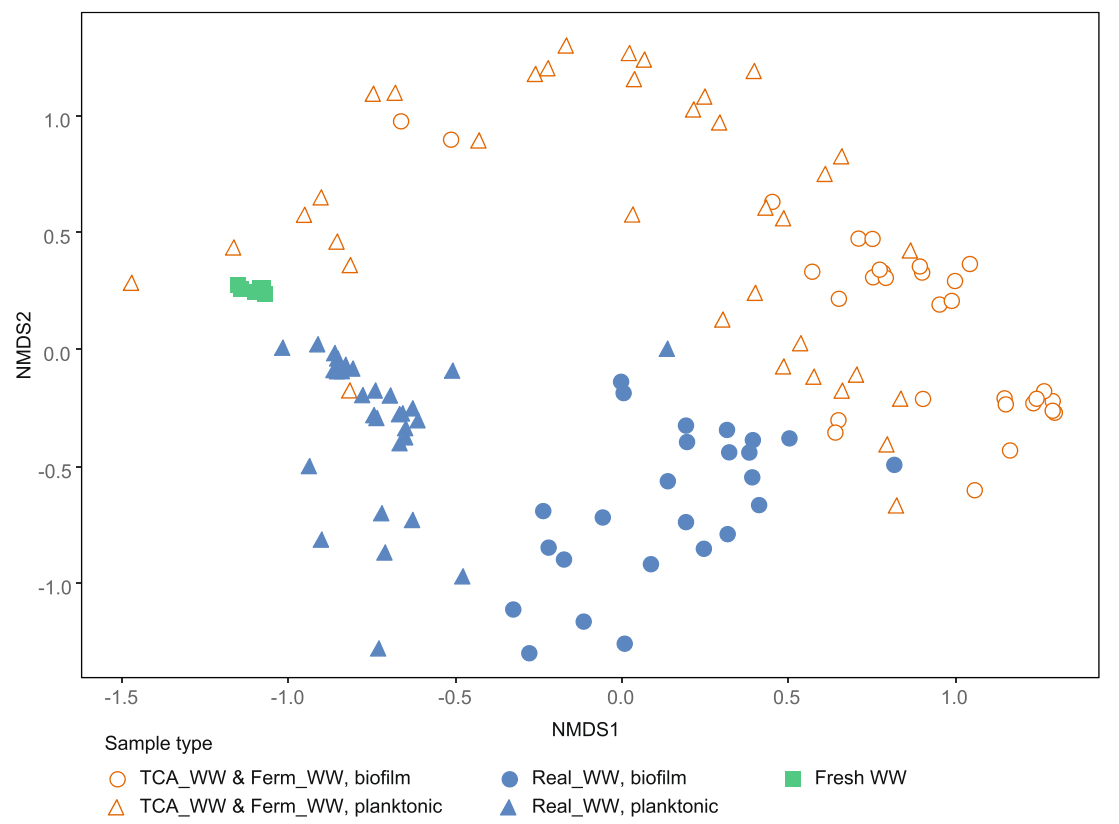

Fig. 2 Bacterial community composition in all samples based on nonmetric multidimensional scaling. The color code of the symbols represents the different reactors running with real (Real_WW) or defined wastewater (TCA_WW and Ferm_WW) as well as the sample origin being the fresh domestic wastewater, biofilm, or planktonic phase. A more detailed assignment of the individual reactors can be found in Supplementary Fig. 1

Nevertheless, also the enrichment of other Deltaproteobacteria (Desulfuromonas up to 50\% in TCA_WW reactor 3, Desulfobulbus up to $1 \%$ ) followed in the subsequent batches, although the inoculation was only performed in batch I. This indicates that the other species also attached to the anode during batch I, but did not increase their abundance as efficient as Geobacter which is well-known for its dominance in electroactive biofilms in similar experiments based on artificial WW. ${ }^{25-27}$ Besides Deltaproteobacteria, also other bacteria contributed to the anode biofilms with a clear difference between TCA_WW and Ferm_WW and for both in comparison with Real_WW (Fig. 3b). The TCA_WW reactors were clearly dominated by Geobacter after batch VI, except TCA_WW 3 showing also a high contribution of Desulfuromonas.

While the acetate oxidation and related current generation is very likely performed by Geobacter as the key player, it is an open question how the oxidation of propionate and butyrate is realized. Geobacter anodireducens has the physiological capacity for propionate utilization if acetate is also present. ${ }^{28}$ In the Ferm_WW reactors, the genus Escherichia/Shigella had a significant contribution with up to $20 \pm 9 \%$ in batch VI. The abundance of this genus was below $1 \%$ in all other reactors indicating its specific role for the sugar fermentation. In the Real_WW reactors, the substrate range is much broader than that in the defined WW reactors. This is also reflected by the community composition of the anodic biofilms showing a high diversity and no dominance of single genera (Fig. 3).

\section{DISCUSSION}

Generally, the planktonic phase of the reactors treating real WW (Real_WW 1-5) was more similar to the inoculum than to the anodic biofilms. This indicates that with the applied anode surface area to reactor volume ratio, the anaerobic electrochemical incubation had only little impact on the composition of the planktonic community. The anodes were immediately colonized within batch I as shown by the current production that sustained for the subsequent batches with anode biofilm communities being clearly different from the initial WW inoculum. These results clearly show that the overall performance in terms of COD degradation and electrochemical activity is mainly governed by the anode biofilms. Nevertheless, alternative reactions like sulfur reduction (see discussion below) or methanogenesis can occur and contribute to COD degradation, thus explaining CE values below $40 \%$.

For elucidating the structure-function relationships, the correlation of anode communities and the overall performance of the reactors were analyzed. While the defined WW reactors (TCA_WW and Ferm_WW) increased their performance from batch I to VI, indicating an adaptation with functional specialization of the microbiome, this performance increase was not observed in the Real_WW reactors. This adaptation with functional specialization in bacterial community composition is mirrored by the diversity of the anode biofilm communities in batch VI (Fig. 3b) but can also be clearly seen when considering all batches. The significantly highest values of the a-diversity indices (Observed genera and Chao1) within the different sample types are found in the fresh WW samples $(1002 \pm 40,1153 \pm 52$, Fig. 4, the complete data set with all values is provided as Supplementary Data 1). Being derived from a WWTP every week, the bacterial community in these samples had probably experienced high variations regarding environmental parameters and daily fluctuations (e.g., COD composition of inflow, weather, etc.) resulting in the variable composition of the bacterial community. At the same time, higher Shannon values in the biofilm $(3.5 \pm 0.48)$ and planktonic $(3.7 \pm$ 0.33) samples of the Real_WW reactors in comparison with the Fresh_WW samples $(2.9 \pm 0.10$, Fig. 4) suggest a more even distribution of the identified bacterial genera in the reactors. Hence, the reactors provide a relatively stable environment and hence a well-defined ecological niche. While in comparison with the Fresh_WW samples, the planktonic samples of Real_WW show only slightly significantly lower values of the a-diversity indices of $902 \pm 78$ (Observed genera) and 1046 \pm 94 (Chao1); the high specialization of the anode biofilm communities to the ecological niche of the anode becomes evident from the highly significantly lower a-diversity values of $765 \pm 49$ (Observed genera) and $910 \pm$ 66 (Chao1), respectively. Furthermore, as the WW is the only source of carbon and electrons in the Real WW reactors, the diversity still has to be relatively high to allow utilization of 

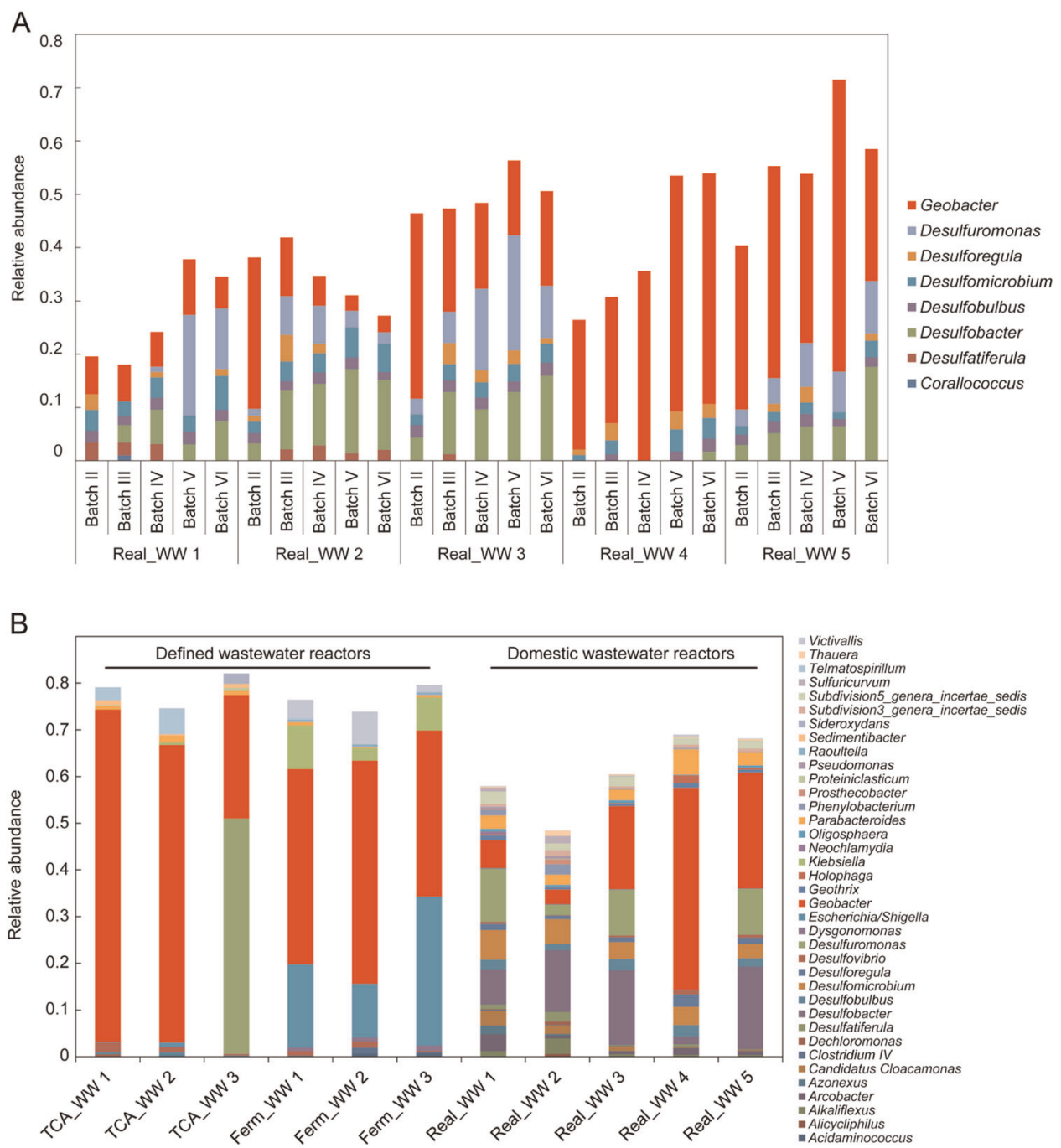

Fig. 3 Bacterial community composition. a Relative abundance of Deltaproteobacteria in the anode biofilms in the Real_WW reactors 1-5 over time. The biofilms formed at the electrode during batch I were not sufficiently dense for sampling and therefore had to be excluded. The relative abundance refers to all sequences in each sample but only genera of Deltaproteobacteria with a contribution of more than $1 \%$ are displayed. b Community composition of the anode biofilms after batch VI, including all genera with an abundance of at least $1 \%$. The defined wastewater reactors TCA_WW 1-3 and Ferm_WW 1-3 were inoculated with 5\% domestic wastewater for batch I. In contrast, Real_WW reactors 1-5 received fresh real WW in each batch as the only source of carbon and repeated inoculum

different and even complex carbon sources. While the electroactive members of the anode biofilm are most likely metabolically limited, as most electroactive species can only utilize simple sugars and small organic acids, ${ }^{14,17}$ the degradation of more complex compounds from the WW has to be performed by other, probably non-electroactive, members of the community.

In contrast, feeding the reactors with defined WW allows a higher degree of adaptation and specialization in the respective biofilms. This specialization goes along with higher functional performance (see Fig. 1) and significantly lower a-diversity values (TCA_WW: $369 \pm 92$ Observed genera, overall $458 \pm 107$ genera according to the Chao1 estimator, Shannon diversity $1.8 \pm 0.62$; Ferm_WW: $343 \pm 60$ Observed genera, $430 \pm 78$ Chao1, and $2.5 \pm$ 0.26 Shannon) compared with the Real_WW biofilms (765 \pm 49 Observed genera, $910 \pm 66$ Chao1, and $3.5 \pm 0.48$ Shannon). The specialization also takes place in the planktonic communities of the defined WW reactors (TCA_WW: $567 \pm 147$ Observed genera, $704 \pm 159$ Chao1, and $2.8 \pm 0.32$ Shannon; Ferm_WW: $483 \pm 121$ Observed genera, $593 \pm 130$ Chao1, and $3.0 \pm 0.40$ Shannon) with a-diversity values being significantly lower than the Real_WW samples (902 \pm 78 Observed genera, $1046 \pm 94$ Chao1, and $3.7 \pm$
0.33 Shannon, Fig. 4). While this specialization is advantageous for the electrochemical performance, it can be a limitation when changes in the WW composition occur and a higher flexibility is required for COD removal and current production (see discussion below).

By comparing the functional performance of all reactors with their bacterial community composition, the key role for the genus Geobacter is obvious (Fig. 3) and in accordance with previous observations, e.g., refs ${ }^{25,29}$.

By considering all reactors, only the abundance of Geobacter shows a correlation with the CE $(r=0.56)$ that is higher than 0.5 (all data given in Table 2). In contrast, the abundance of Geobacter does not significantly correlate to the current production and COD removal although positive trends between Geobacter abundance and performance parameters can be seen for the individual reactors. For the combined data set of all reactors, the abundance of several other genera possesses a correlation higher than 0.5 (Table 2). When considering only the data of the biofilms from the Real_WW reactors, the correlation between CE and Geobacter is less pronounced and also other taxa contribute to the same (Supplementary Table 2). This supports the specific role of 


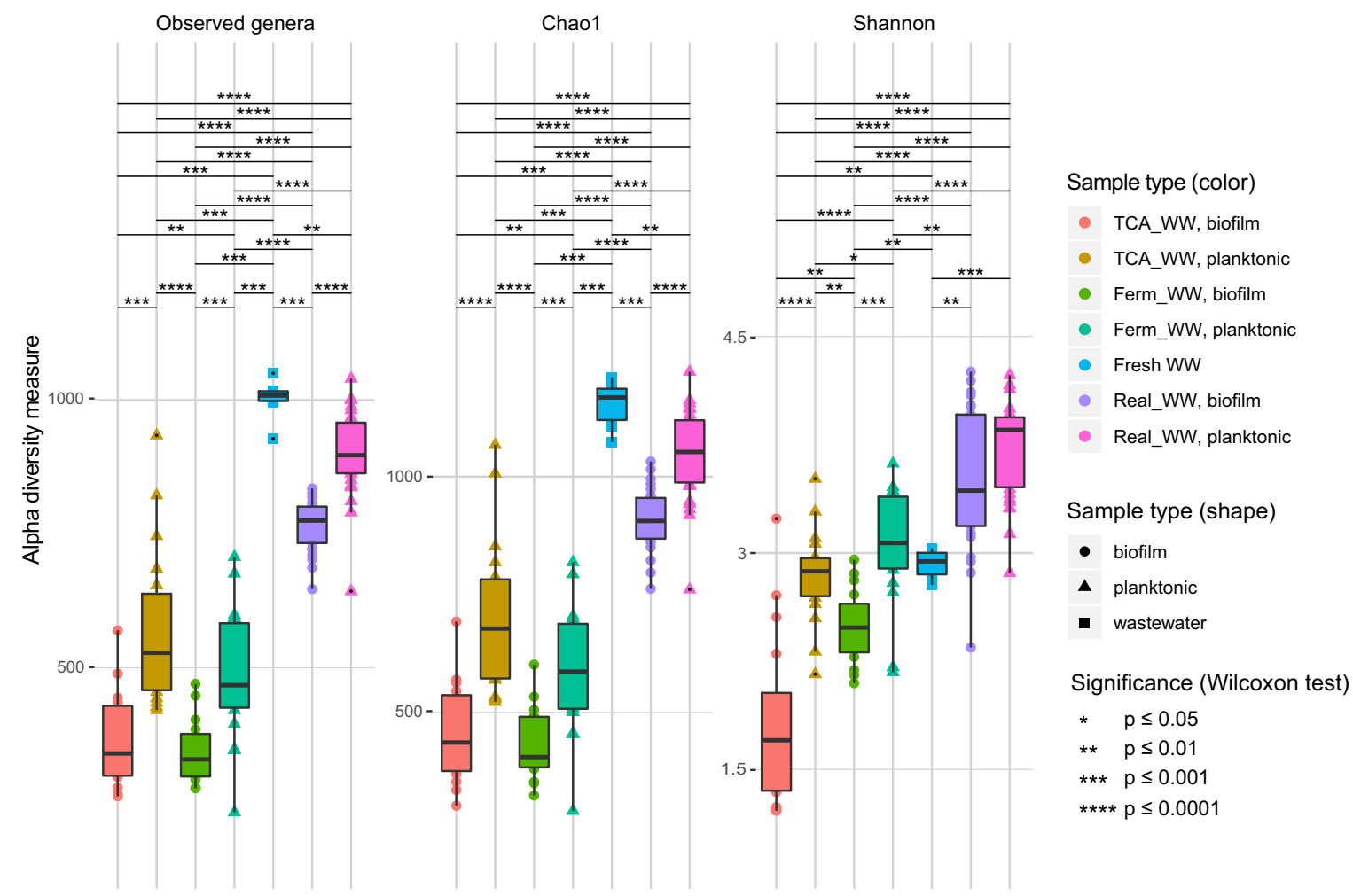

Fig. $4 \alpha$-Diversity indices in all samples based on the diversity measures Observed genera, Chao1, and Shannon. The color code of the symbols represents the different reactors running with real (Real_WW) or defined wastewater (TCA_WW and Ferm_WW). The shape of the symbols is referring to the sample origin, the fresh domestic wastewater (square), biofilm (circle), or planktonic phase (triangle). The calculation of $p$ values was performed with the R packages vegan, iNext, ${ }^{27}$ and RDPutils ${ }^{23}$

Table 2. Correlation analysis of all reactors: positive and negative correlations of the relative abundance of microbial genera with reactor performance parameters considering the complete data set with all reactors (Real_WW, Ferm_WW, and TCA_WW)

\begin{tabular}{lcll}
\hline & $q$ & CE & COD removal \\
\hline Acidaminococcus & 0.79 & & \\
Akkermansia & & & 0.50 \\
Anaerovorax & & 0.59 \\
Bacteroides & 0.55 & & \\
Clostridium_IV & 0.53 & & \\
Desulfobulbus & -0.51 & & \\
Dialister & & & \\
Escherichia/Shigella & 0.58 & & 0.51 \\
Gemmiger & & & \\
Geobacter & & 0.56 & 0.52 \\
Geothrix & & & \\
Phascolarctobacterium & 0.72 & & \\
\hline
\end{tabular}

Only values above 0.5 (positive correlation) and below -0.5 (negative correlation) are shown

Geobacter for the efficiency in terms of current production from oxidation of small organic acids. The degradation of more complex organic compounds depends on other microbial community members that vary for the respective reactors and depend on the provided substrates as well as stochastic events as, e.g., the difference in the presence of low abundant microorganisms during inoculation and colonization.

When now analyzing the potential interactions among the bacteria based on microbiome network analysis (Fig. 5), Geobacter is very surprisingly absent from the network covering 74 out of 231 genera. The most important clusters in terms of performance are clusters I and V. Most members of these clusters show a positive correlation to the performance parameters (Supplementary Table 2 ) and in addition cluster $\mathrm{V}$ relates positively to sulfide production being in accordance with the higher sulfide concentration in the Real WW reactors 3-5. While the increase in sulfide due to sulfur reduction feels counterintuitive for the successful anode respiration, the respective taxa might have the capacity to perform both anode respiration as well as sulfur reduction. In contrast, the members in cluster II including, e.g., Thiobacillus and Rhizobium, show mainly negative correlations to the bioelectrochemical WW degradation in terms of CE and $j$. Hence, they are probably involved in COD degradation pathways (positive correlation for COD) independent from the anode. This could explain the lower performance of Real_WW reactor 1 as here these genera had higher contributions to the anodic biofilm community than those in the other Real_WW reactors. Other clusters like clusters III and IV that also contain potential disease-associated genera (Enterobacter, Salmonella, and Legionella) seem independent from the reactor performance. Yet, they show a clear cooccurrence that is, e.g., related to the presence of sucrose in the Ferm_WW reactors 1-3 for cluster III.

While being present in all biofilms, the absence of the genus Geobacter from the network means that it does not show a distinct correlation with one or a few other genera over all anode biofilms. This supports previous observations and the specific ecological role, as well as the outstanding functionality of Geobacter in anode biofilms. Geobacter is the fastest and most competitive primary 


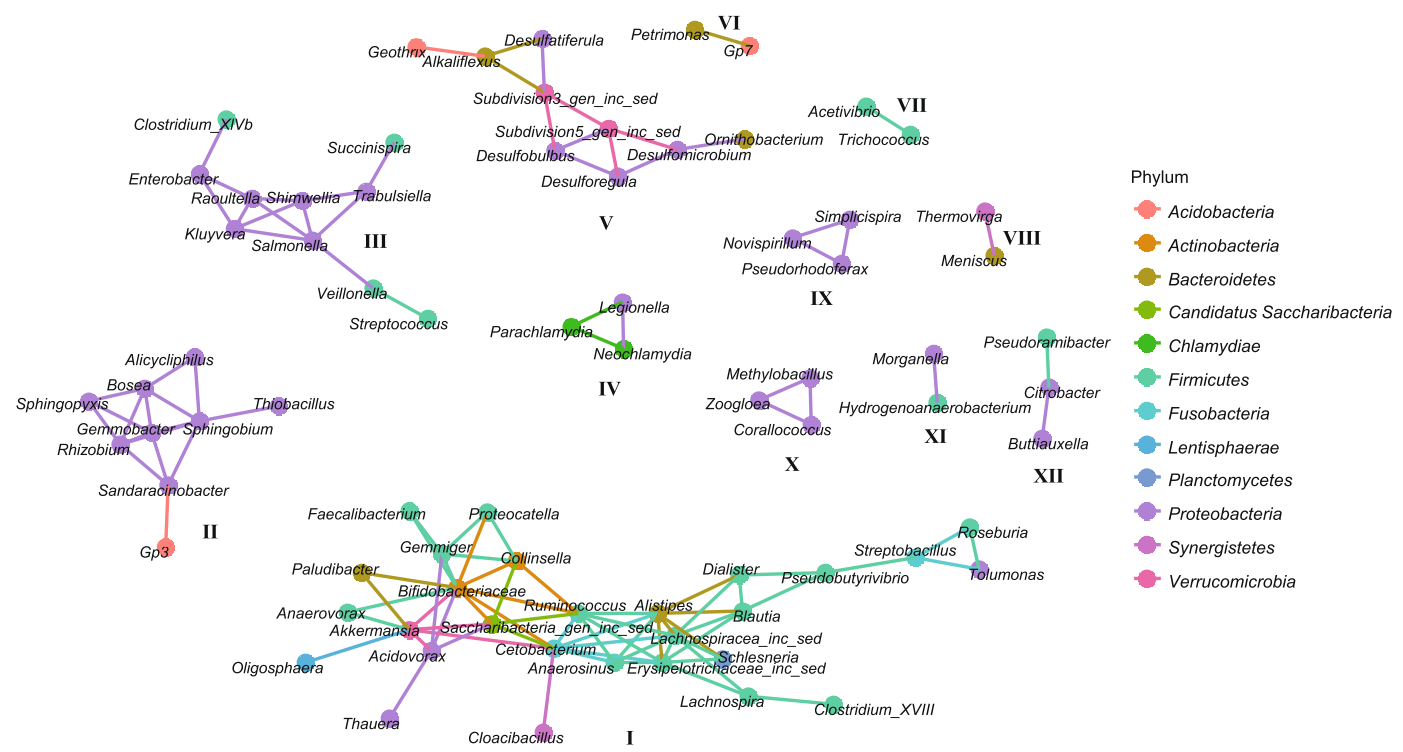

Fig. 5 Microbiome interaction network. Interestingly, as a result of this analysis, Geobacter is not included; thus, it is not connected to the other genera. This supports the hypothesis that Geobacter occupies a specific ecological niche within the anode biofilm independent from general COD degradation and other microorganisms. The biggest cluster (I) has the highest importance for the functional performance of the reactors followed by cluster $\mathrm{V}$, while the members of cluster II show negative correlations to reactor performance parameters and are probably responsible for alternative COD degradation pathways being independent of anode respiration

colonizer of the provided ecological niche being an anode surface serving as TEA. However, Geobacter is not able to degrade complex substrates as those usually found in WW. Thus, other members being part of the planktonic community (e.g., those in cluster I) or the biofilm (e.g., those in cluster V) are required to develop a trophic network allowing COD degradation as well as current production. Thus, there is no cluster including typically known electroactive microorganisms. ${ }^{17}$ Therefore, the question persists, if Geobacter as the successful first colonizer remains also the only electroactive taxon in these biofilms over time, or if other taxa either using direct or mediated EET also contribute to current generation. Altogether, the microbiome network analysis leads to the conclusion that it is not an individual genus that is governing the reactor performance. Rather, the diversity and complexity of all members-and their division of labor-within the trophic network is of functional importance with Geobacter driving the reactor performance regarding $C E$.

The initial colonization of an anode surface is very likely to be performed by Geobacter anodireducens together with lessabundant species. Over the course of WW treatment, these species can increase their abundance and adapt the biofilm composition in a flexible way toward the specific substrates provided as well as other important ecological parameters like temperature, $\mathrm{pH}$, the presence of alternative electron acceptors, etc. $^{30}$ While pre-enrichment of Geobacter on anodes is often performed in laboratory reactors (e.g., refs ${ }^{31,32}$ ), this aspect is usually not considered for the other microbial community members, which makes their contribution to electrochemical performance and COD removal rather stochastic.

What does this now mean for the implementation of MET for WW treatment? Generally, reactor microbiomes that are less diverse and more stable in terms of taxon composition can provide the highest performance under fixed conditions (i.e., highly reproducible conditions and WW composition, e.g., specific industrial WW). This is also the case for microbial anodes that are usually achieved by pre-enrichment from real WW using acetate as the only substrate. This is certainly highly suitable for fundamental studies and can also be adopted for technical implementation in case the targeted WW contains acetate or a COD that can be easily converted therein. The secondary colonization by the microorganisms providing the acetate from COD, will then be realized by the natural community of the WW. But one has to keep in mind that such a high specialization of a microbial biofilm is very sensitive to any change of the growth conditions which can result in significant functional losses up to complete biofilm detachment (see e.g., ref. ${ }^{31}$ ). Most WW is less stable in its composition and environmental conditions are usually fluctuating. In these cases, colonization of the pre-enriched anode within the final habitat will result in a higher variability regarding anode biofilm composition. Most likely, functional anodic biofilms will be formed, but there is still the chance that the primary colonization, e.g., by Geobacter anodireducens, is not the optimum for the WW to be treated. Here, a specific recommendation is case dependent. One option would be to pre-colonize the anode with a very diverse microbial inoculum and use of a highly diverse substrate (being representative of the average WW to be treated, see e.g., ref. ${ }^{33}$ ). Once a diverse anode biofilm is established, it can be transferred to the site of application. Also here, realistic abiotic conditions should be applied for the pre-enrichment to allow a transfer to practice. In conclusion, the specific composition and variability of the WW to be treated has to be accounted for optimal process development under consideration of general ecological principles. ${ }^{30}$ Therefore, we assume WW of more constant composition, e.g., from food industries, being more likely to be treated using MET than municipal WW.

\section{METHODS}

Chemicals, potential reference, and WWs

All chemicals were of analytical or biochemical grade. All potentials provided refer to the SHE by conversion from $\mathrm{Ag} / \mathrm{AgCl}$ (sat. $\mathrm{KCl},+197 \mathrm{mV}$ vs. SHE).

Three types of WW were used in order to address three different metabolic capacities and hence trophic levels that are relevant for successful COD degradation: two defined WWs allowing either only tricarboxylic acid (TCA) cycle (TCA_WW) or allowing TCA and glycolysis (Ferm_WW), and real domestic WW (Real_WW). For better comparison, the charge, coulombic efficiency, and the removal of organic compounds (in all reactors monitored in terms of COD equivalents) are given in Supplementary Table 1. In addition, the chemical composition was 
investigated in detail for the reactors run with TCA_WW and Ferm_WW using HPLC.

TCA_WW and Ferm_WW were based on a carbonate-buffered mineral medium ${ }^{29}$ that contained $30 \mathrm{mM}$ of the carbon source equaling a COD of $0.6 \mathrm{~g} \mathrm{~L}^{-1}$ in both cases. TCA_WW contained $3.75 \mathrm{mM}$ sodium propionate, $3.75 \mathrm{mM}$ sodium butyrate, and $2 \mathrm{mM}$ sodium acetate. Ferm_WW contained $2 \mathrm{mM}$ sucrose, $1 \mathrm{mM}$ sodium propionate, $1 \mathrm{mM}$ sodium butyrate, and $1 \mathrm{mM}$ sodium acetate. The Real_WW had an average COD of $473 \pm 74 \mathrm{mg} \mathrm{L}^{-1}$.

Fresh WW was obtained weekly from the primary clarifier (after passing mechanical filtration) of the municipal WWTP (Abwasserzweckverband für die Reinhaltung der Parthe, Am Klärwerk, 04451 Borsdorf), Germany. If not used immediately, it was stored at $4{ }^{\circ} \mathrm{C}$ for a maximum of $24 \mathrm{~h}$. Prior to usage, the solids in the WW were resuspended by shaking. The fresh WW served as the sole growth medium and inoculum for the Real_WW reactors and was used as inoculum for TCA_WW and Ferm_WW reactors by adding $25 \mathrm{~mL}$ in a $500-\mathrm{mL}$ reactor at the beginning of the experiments (i.e., batch I, see also below).

\section{Experimental setup}

MET reactors were based on tailor-made glass reactors ${ }^{34}$ and shown in Supplementary Fig. 2. Working electrodes (WE) and counter electrodes were made from graphite rods and plates (CP-2200 quality, CP-Handels $\mathrm{GmbH}$, Wachtberg, Germany). The WE and counter electrodes had 47.6and $15 \mathrm{~cm}^{2}$ geometric surface area, respectively, and were separated by a membrane (fumasep ${ }^{\circledR}$ FKE, Fumatech $\mathrm{GmbH}$, Bissingen, Germany). WE chambers contained $500 \mathrm{~mL}$ of WW leading to an anodic surface-area-tovolume ratio of $95.2 \mathrm{~cm}^{2} \mathrm{~L}^{-1}$ at the beginning and $76 \mathrm{~cm}^{2} \mathrm{~L}^{-1}$ at the end of the experiment, i.e., after six batches of operation (due to sampling, see below). Counter electrode chambers were filled with $10 \mathrm{~mL}$ of a phosphate buffer $\left(1.8 \mathrm{~g} \mathrm{~L}^{-1} \mathrm{Na}_{2} \mathrm{HPO}_{4}, 0.223 \mathrm{~g} \mathrm{~L}^{-1} \mathrm{NaH}_{2} \mathrm{PO}_{4}\right.$, and $8.5 \mathrm{~g} \mathrm{~L}^{-1} \mathrm{NaCl}, \mathrm{pH}$ 7.2). Reference electrodes (Ag/AgCl sat. $\mathrm{KCl}, \mathrm{SE} \mathrm{11}$, Meinsberg Sensortechnik $\mathrm{GmbH}$, Germany) were introduced in the WE chamber. WE were poised at $+397 \mathrm{mV}$ vs. SHE with recording of current every $10 \mathrm{~min}$ by using a multipotentiostat (MPG-2, Bio-Logic SAS, Claix, France). Note that the potentiostatic operation assured that differences in the electrolytic conductivity did not have an impact on the potential of the WE.

Fresh WW was introduced in the WE chambers and purged with nitrogen for $25 \mathrm{~min}$. The experiments were conducted at room temperature and stirring at $150 \mathrm{rpm}$ by using magnetic stirrers. Three independent replicate reactors were run for each TCA_WW and Ferm_WW and five independent replicate reactors for Real_WW. All reactors were started and run in parallel.

The experiment was run over six batches, which were labeled accordingly as batches I-VI, for a duration of 7 days per batch. The time for each batch was set constant to 7 days in order to synchronize the development of the reactors without facing starvation periods. After each batch, the WW of all reactors was completely exchanged and liquid samples of the treated WW (planktonic phase) and biofilm samples of the WE were collected. The WE was sampled by cutting a defined piece of the plate ( $1 \mathrm{~cm}$ length and hence $2.4 \mathrm{~cm}^{2}$ surface area, Supplementary Fig. $2 \mathrm{~B}$ ). Subsequently, fresh WW was introduced in the anode chambers and purged with nitrogen for $25 \mathrm{~min}$. The TCA_WW and Ferm_WW reactors received the respective sterile WW without any inoculate in batches II-VI. Each batch the cathode chamber was also refilled with new buffer solution. Chemical analysis (COD, TOC, sulfate, total nitrogen, nitrate, ammonium, phosphate, conductivity, $\mathrm{pH}$ of the fresh and treated real_WW, HPLC analysis of sucrose, acetate, propionate, and $n$-butyrate for TCA_WW and Ferm_WW) was performed using standard methods (details are given in Supplementary methods).

\section{DNA extraction}

The cut piece of the WE was stored at $-20^{\circ} \mathrm{C}$ until DNA extraction. In total, $3 \mathrm{~mL}$ of treated WW were centrifuged for $10 \mathrm{~min}$ with $14,000 \mathrm{~g}$ at $4^{\circ} \mathrm{C}$. The supernatant was removed and the pellet stored at $-20^{\circ} \mathrm{C}$ until DNA extraction. DNA was extracted using the NucleoSpin ${ }^{(R)}$ Tissue Kit (Macherey Nagel, Düren, Germany). The WW pellet was redissolved in the first extraction buffer containing $\mathrm{T} 1$ + Proteinase $\mathrm{K}$. The same buffer was also added to the WE piece. All samples were incubated at $56^{\circ} \mathrm{C}$ for $2 \mathrm{~h}$ and then further processed according to the manufacturer's recommendation.
Amplicon preparation, next-generation sequencing, and amplicon analysis pipeline

High-throughput sequencing of the 16S rRNA gene (V3 region) was applied to determine bacterial community composition of the fresh domestic WW, the treated WW, and the anodic biofilm samples. A preamplification of the $\mathrm{V} 3$ region of the 16S rRNA gene (primer pair 341f-515r) was followed by amplicon preparation as described by Bartram et al. ${ }^{35}$ Amplicons were sequenced on the Illumina NextSeq550 platform (San Diego, CA, USA) in 150-bp pair-end mode and generated a total of $334,203,639$ bacterial sequences which were quality-checked by the FastQC program version 0.10.1 (Simon Andrews; http://www. bioinformatics.babraham.ac.uk/projects/fastqc/). Due to quality loss at the ends of forward and reverse reads, those were trimmed to a length of $130 \mathrm{bp}$. A JAVA program DimerFilter based on FastQC removed potential primer dimers from the raw sequence data. Forward and the reverse reads were joined by fastq-join ${ }^{36}$ using a $20 \%$ mismatch and a minimum overlap of $6 \mathrm{bp}$. Result files were converted to FASTA and checked for chimeras with Uchime (Usearch 5.2.32 $2^{37}$ ) against the gold database provided by ChimeraSlayer (http://drive5.com/otupipe/gold.tz). Finally, RDP classifier version $2.10 .1^{38,39}$ was applied for taxonomic-dependent analysis employing a confidence value of 0.5 , as recommended for short-read amplicon data.

\section{Data analysis and statistics}

The current density $(j)$ is provided per geometric surface area and the Coulombic efficiency (CE) was calculated based on the COD equivalents (see also Supplementary Table 3). The experimental data were analyzed with Excel and Origin and the phylogenetic data with $\mathrm{R}$ using the package phyloseq. $^{40}$ The presented data give the average \pm standard deviation of three independent biological replicates for the TCA_WW and Ferm_WW reactors and of five independent biological replicates of the Real_WW reactors. Where appropriate, the individual data of each reactor are shown. Correlation analysis was based on Spearman's rank correlation coefficient using the $R$ package vegan. ${ }^{41}$ The calculation of a-diversity values and the rarefaction curves (Supplementary Fig. 3) was performed with the $\mathrm{R}$ packages vegan, iNext, ${ }^{42}$ and RDPutils. ${ }^{38}$

\section{Reporting summary}

Further information on research design is available in the Nature Research Reporting Summary linked to this article.

\section{DATA AVAILABILITY}

The data that support the findings of this study are available from the corresponding author upon reasonable request.

Received: 8 April 2019; Accepted: 2 September 2019; Published online: 27 September 2019

\section{REFERENCES}

1. Timmis, K. et al. The contribution of microbial biotechnology to economic growth and employment creation. Microb. Biotechnol. 10, 1137-1144 (2017).

2. Nielsen, P. H., Raunkjær, K., Norsker, N. H., Jensen, N. A. \& Hvitved-Jacobsen, T. Transformation of wastewater in sewer systems-a review. Water Sci. Technol. 25, 17-31 (1992).

3. Korth, B., Maskow, T., Günther, S. \& Harnisch, F. Estimating the energy content of wastewater using combustion calorimetry and different drying processes. Front. Energy Res. 5, 23 (2017).

4. Logan, B. E. Exoelectrogenic bacteria that power microbial fuel cells. Nat. Rev. Microbiol. 7, 375-381 (2009).

5. Kumar, A. et al. The ins and outs of microorganism-electrode electron transfer reactions. Nat. Rev. Chem. 1, 0024 (2017).

6. Lovley, D. R. Syntrophy goes electric: direct interspecies electron transfer. Ann. Rev. Microbiol. 71, 643-664 (2017).

7. Schröder, U., Harnisch, F. \& Angenent, L. T. Microbial electrochemistry and technology: terminology and classification. Energy Environ. Sci. 8, 513-519 (2015).

8. Logan, B. E. \& Rabaey, K. Conversion of wastes into bioelectricity and chemicals by using microbial electrochemical technologies. Science 337, 686-690 (2012).

9. Heidrich, E. S., Edwards, S. R., Dolfing, J., Cotterill, S. E. \& Curtis, T. P. Performance of a pilot scale microbial electrolysis cell fed on domestic wastewater at ambient temperatures for a 12 month period. Biores. Technol. 173, 87-95 (2014). 
10. Brown, R. K. et al. Evaluating the effects of scaling up on the performance of bioelectrochemical systems using a technical scale microbial electrolysis cell. Biores. Technol. 163, 206-213 (2014).

11. Lovley, D. R. Electromicrobiology. Ann. Rev. Microbiol. 66, 391-409 (2012).

12. Bond, D. R. \& Lovley, D. R. Electricity production by geobacter sulfurreducens attached to electrodes. Appl. Environ. Microbiol. 69, 1548-1555 (2003).

13. Bretschger, O. et al. Current production and metal oxide reduction by Shewanella oneidensis MR-1 wild type and mutants. Appl. Environ. Microbiol. 73, 7003-7012 (2007).

14. Kiely, P. D., Regan, J. M. \& Logan, B. E. The electric picnic: synergistic requirements for exoelectrogenic microbial communities. Curr. Opin. Biotechnol. 22, 378-385 (2011).

15. Marsili, E. et al. Shewanella secretes flavins that mediate extracellular electron transfer. Proc. Natl Acad. Sci. USA 105, 3968-3973 (2008).

16. Federal Ministry for the Environment, Nature Conservation and Nuclear Safety, Germany. Waste Water Ordinance. http://www.bmu.de/fileadmin/bmu-import/ files/pdfs/allgemein/application/pdf/wastewater_ordinance.pdf (2004).

17. Koch, C. \& Harnisch, F. Is there a specific ecological niche for electroactive microorganisms? ChemElectroChem 3, 1282-1295 (2016).

18. Rosa, L. F. M., Koch, C., Korth, B. \& Harnisch, F. Electron harvest and treatment of amendment free municipal wastewater using microbial anodes: a case study. J. Power Sources 356, 319-323 (2017).

19. Zhou, J. et al. Stochastic assembly leads to alternative communities with distinct functions in a bioreactor microbial community. mBio 4, 2 (2013).

20. Ofițeru, I. D. et al. Combined niche and neutral effects in a microbial wastewater treatment community. Proc. Natl Acad. Sci. USA 107, 15345-15350 (2010).

21. Koch, C., Popiel, D. \& Harnisch, F. Functional redundancy of microbial anodes fed by domestic wastewater. ChemElectroChem 1, 1923-1931 (2014).

22. Kim, H. M., Hwang, C. Y. \& Cho, B. C. Arcobacter marinus sp. nov. Int J. Syst. Evol. Microbiol. 60, 531-536 (2010).

23. Sasi Jyothsna, T. S., Rahul, K., Ramaprasad, E. V. V., Sasikala, C. \& Ramana, C. V. Arcobacter anaerophilus sp. nov., isolated from an estuarine sediment and emended description of the genus Arcobacter. Int J. Syst. Evol. Microbiol. 63 4619-4625 (2013).

24. Bergey's Manual of Systematic Bacteriology. The Proteobacteria. Part C The AlphaBeta-, Delta-, and Epsilonproteobacteria. (Springer, New York, USA, 2005). Volume Two.

25. Torres, C. I. et al. Selecting anode-respiring bacteria based on anode potential: phylogenetic, electrochemical, and microscopic characterization. Environ. Sci. Technol. 43, 9519-9524 (2009).

26. Cusick, R. D., Kiely, P. D. \& Logan, B. E. A monetary comparison of energy recovered from microbial fuel cells and microbial electrolysis cells fed winery or domestic wastewaters. Int J. Hydrog. Energy 35, 8855-8861 (2010).

27. Harnisch, F. et al. Revealing the electrochemically driven selection in natural community derived microbial biofilms using flow-cytometry. Energy Environ. Sci. 4, 1265-1267 (2011).

28. Sun, D., Wang, A., Cheng, S., Yates, M. \& Logan, B. E. Geobacter anodireducens sp. nov., an exoelectrogenic microbe in bioelectrochemical systems. Int J. Syst. Evol. Microbiol. 64, 3485-3491 (2014).

29. Ishii, Si et al. Microbial population and functional dynamics associated with surface potential and carbon metabolism. ISME J. 8, 963-978 (2014).

30. Koch, C., Korth, B. \& Harnisch, F. Microbial ecology-based engineering of microbial electrochemical technologies. Microb. Biotechnol. 11, 22-38 (2018).

31. Madjarov, J., Prokhorova, A., Messinger, T., Gescher, J. \& Kerzenmacher, S. The performance of microbial anodes in municipal wastewater: pre-grown multispecies biofilm vs. natural inocula. Biores. Technol. 221, 165-171 (2016).

32. Miceli, J. F., Parameswaran, P., Kang, D.-W., Krajmalnik-Brown, R. \& Torres, C. I. Enrichment and analysis of anode-respiring bacteria from diverse anaerobic inocula. Environ. Sci. Technol. 46, 10349-10355 (2012).

33. Riedl, S. et al. Successive conditioning in complex artificial wastewater increases the performance of electrochemically active biofilms treating real wastewater. ChemElectroChem 4, 3081-3090 (2017).

34. Koch, C. et al. Coupling electric energy and biogas production in anaerobic digesters - impacts on the microbiome. RSC Adv. 5, 31329-31340 (2015).

35. Bartram, A. K., Lynch, M. D. J., Stearns, J. C., Moreno-Hagelsieb, G. \& Neufeld, J. D. Generation of multimillion-sequence 16S rRNA gene libraries from complex microbial communities by assembling paired-end illumina reads. Appl Environ. Microbiol. 77, 3846-3852 (2011).
36. Aronesty, E. Comparison of sequencing utility programs. Open Bioinf. J. 7, 1-8 (2013).

37. Edgar, R. C., Haas, B. J., Clemente, J. C., Quince, C. \& Knight, R. UCHIME improves sensitivity and speed of chimera detection. Bioinformatics 27, 2194-2200 (2011).

38. Cole, J. R. et al. Ribosomal Database Project: data and tools for high throughput rRNA analysis. Nucleic Acids Res. 42, D633-D642 (2014)

39. Wang, Q., Garrity, G. M., Tiedje, J. M. \& Cole, J. R. Naïve Bayesian classifier for rapid assignment of rRNA sequences into the new microbial taxonomy. Appl. Environ. Microbiol. 73, 5261-5267 (2007).

40. McMurdie, P. J. \& Holmes, S. phyloseq: an R package for reproducible interactive analysis and graphics of microbiome census data. PLOS ONE 8, e61217 (2013).

41. Oksanen, J. et al. Community Ecology Package "vegan". https://CRAN.R-project. org/package = vegan (2018).

42. Hsieh, T. C., Ma, K. H., Chao, A. iNEXT: iNterpolation and EXTrapolation for species diversity. $\mathrm{R}$ package version 2.0.17. http://chao.stat.nthu.edu.tw/blog/softwaredownload/ (2018).

\section{ACKNOWLEDGEMENTS}

The authors thank Desiree Schmidt (UFZ) for laboratory assistance and Alicia Geppert (DSMZ) for the preparation of the V3 amplicon libraries. FH acknowledges support by the BMBF (Research Award "Next generation biotechnological Processes-Biotechnology 2020+") and the Helmholtz Association (Young Investigators Group). This work was supported by the Helmholtz Association within the Research Program Renewable Energies.

\section{AUTHOR CONTRIBUTIONS}

The study was designed and performed by C.K. and F.H. including conception, experimental design, data acquisition, interpretation of data, and paper preparation. $\mathrm{KJH}, \mathrm{BB}$, and $\mathrm{JO}$ performed sequencing and the respective data preparation and paper editing. All authors have read and approved the paper.

\section{COMPETING INTERESTS}

The authors declare no competing interests.

\section{ADDITIONAL INFORMATION}

Supplementary information is available for this paper at https://doi.org/10.1038/ s41522-019-0100-y.

Correspondence and requests for materials should be addressed to C.K. or F.H.

Reprints and permission information is available at http://www.nature.com/ reprints

Publisher's note Springer Nature remains neutral with regard to jurisdictional claims in published maps and institutional affiliations.

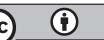

Open Access This article is licensed under a Creative Commons Attribution 4.0 International License, which permits use, sharing, adaptation, distribution and reproduction in any medium or format, as long as you give appropriate credit to the original author(s) and the source, provide a link to the Creative Commons license, and indicate if changes were made. The images or other third party material in this article are included in the article's Creative Commons license, unless indicated otherwise in a credit line to the material. If material is not included in the article's Creative Commons license and your intended use is not permitted by statutory regulation or exceeds the permitted use, you will need to obtain permission directly from the copyright holder. To view a copy of this license, visit http://creativecommons. org/licenses/by/4.0/.

(c) The Author(s) 2019 